\title{
An uncomplicated electrochemical sensor combining a perfluorocarbon SAM and ACE2 as the bio-recognition element to sensitively and specifically detect SARS-CoV-2 in complex samples.
}

\author{
Vincent J Vezza ${ }^{a}$, Adrian Butterwortha, Perrine Lasserrea, Ewen 0 Blair ${ }^{\mathrm{a}}$, Alexander \\ MacDonalda, Stuart Hannaha, Christopher Rinaldib Paul A Hoskissonc, Andrew C Wardd, \\ Alistair Longmuire, Steven Setforde, Michael E Murphyf,g, Damion K Corrigana.
}

\author{
a - Department of Biomedical Engineering, University of Strathclyde, Glasgow, UK, G1 1XP. \\ b - Department of Pure \& Applied Chemistry, University of Strathclyde, Glasgow, UK, G1 1XP \\ c - Strathclyde Institute of Pharmacy and biomedical Sciences (SIPBS), University of Strathclyde, Glasgow, UK, G1 1XP \\ d - Department of Civil and Environmental Engineering, University of Strathclyde, Glasgow, UK, G1 1XP \\ e - LifeScan Scotland Ltd, Beechwood Park North, Inverness UK, IV2 3ED \\ f - NHS GGC, Glasgow Royal Infirmary, Department of Microbiology, NEW Lister Building, Glasgow (UK) G31 2ER \\ g- School of Medicine, Dentistry \& Nursing, College of Medical Veterinary \& Life Sciences, University of Glasgow, G12 \\ 8QQ
}

\begin{abstract}
Emerging in late 2019, the SARS-CoV-2 virus has had devastating health and economic effects around the world forcing governments to enact restrictions on day to day life, resulting in severe economic and social disruption. The virus has stimulated new research in the fields of drug development, vaccinology and diagnostic testing. Here, we present the basis for a simple, mass manufacturable saliva based electrochemical assay for the SARS-CoV-2 virus achieved through adsorption of the Angiotensin Converting Enzyme 2 (ACE2) into a thiolated amphiphobic perfluoro monolayer assembled on a gold sensor surface. Following sensor preparation, it is possible to measure specific binding of recombinant spike protein and discriminate positive and negative samples of inactivated SARS-CoV-2 following 30 minutes incubation under ambient conditions. Representative calculations of limits of detection are made for recombinant spike proteins $(1.68 \mathrm{ng} / \mathrm{mL})$ and inactivated virus $(37.8 \mathrm{dC} / \mathrm{mL})$. The assay as presented ultimately shows discrimination between positive and negative inactivated SARS-CoV-2 samples originating from a clinical molecular standards kit intended for clinical and biomedical assay validation, and which is designed to mimic clinical samples through presence of cells and proteins in the sample medium. The simple design of the label free measurement and the selection of reagents involved means the assay has clear potential for transfer onto mass producible units such as screen-printed electrodes similar to glucose-format test strips, to enable widespread, low cost and rapid testing for SARS-CoV-2 in the general population.
\end{abstract}

Keywords: electrochemical sensors, electrochemical impedance spectroscopy (EIS), SARS-CoV-2, COVID-19. 


\section{Introduction}

SARS-CoV-2 came to the attention of health authorities in China during late 2019 and was followed by a declaration of a "public health emergency of international concern" on the $30^{\text {th }}$ of January 2020. SARS-CoV-2 went on to quickly spread around the globe and was declared a pandemic by the World Health Organisation on the $11^{\text {th }}$ of March (World Health Organisation, 2020a, 2020b). The virus itself, SARS-CoV-2, is the aetiological agent of coronavirus disease (COVID-19). Diagnostics have been a major challenge thus far in the pandemic with assay quantity, reagent costs and time to result being of prime concern. The virion is between 50 and $200 \mathrm{~nm}$ in diameter and has four major structural proteins (Masters, 2006). One of these proteins, the "spike" protein, is responsible for binding to the surface of cells which are known to express angiotensin converting enzyme 2 (ACE2) on their surface. The affinity between ACE2 and the spike protein of SARS-CoV-2 has been shown to be in the low nanomolar range (Lan et al., 2020). Due to this high affinity between the spike protein and ACE2 it is possible for ACE2 to be deployed as a receptor in various biosensor formats. A feature of the ACE2 enzyme of particular importance is the hydrophobic region which normally facilitates insertion into the cell membrane (Warner et al., 2004). This allows ACE2 to be inserted into synthetically made amphiphobic structures, which resemble cell membranes.

Electrochemical biosensors are a promising route to realising rapid and sensitive detection of a large range of pathogens and clinically important biomarkers (Cesewski and Johnson, 2020; Mehrotra, 2016). The most wellknown example is the glucose biosensor (most commonly an amperometric sensor), which is in widespread use for the home testing of blood glucose levels and which serves diabetic patients so well in the routine monitoring of blood sugar levels (Newman and Setford, 2006). Numerous other biosensors have been developed which operate by a range of principles (Turner, 2013), including, cyclic voltammetry (CV) (Manzano et al., 2018; Tancharoen et al., 2019), linear sweep voltammetry (LSV) (Shaibani et al., 2017; Tong et al., 2013), electrochemical impedance spectroscopy (EIS) (Butterworth et al., 2019; Jiang et al., 2017) and differential pulse voltammetry (DPV) (Blair et al., 2020; Kim et al., 2010). EIS involves a measurement setup where a small AC excitation potential is imposed at the working electrode (often under open circuit potential) and the resulting current response of the electrochemical cell is measured. Various parameters associated with the cell and its response can be extracted from the EIS response, and these include the solution resistance (Rs), the double layer capacitance $\left(\mathrm{C}_{\mathrm{DL}}\right)$, the charge transfer resistance $\left(\mathrm{R}_{\mathrm{ct}}\right)$ and the Warburg impedance $(\mathrm{W})$ (Bard and Faulkner, 2001). The double layer capacitance and the charge transfer resistance have been shown to be particularly effective for the label-free monitoring of binding at biologically functionalised electrode surfaces. These techniques enable the sensitive and specific measurement of DNA and protein biomarkers which has been shown repeatedly in the literature (Bahadır and Sezgintürk, 2016; Lisdat and Schäfer, 2008).

For many electrochemical biosensors, like those described above, surface functionalisation and attachment chemistry play a major role in sensor design and performance (Guo et al., 1994; Yang et al., 1995). For gold sensors, the attachment of biological molecules often takes place through use of gold-thiol attachment and more specifically through the formation of self-assembled monolayers (SAMs) (Bain et al., 1989). SAMs serve the dual purpose of blocking the electrode surface from non-specific binding of proteins, cells and other components in a sample medium and can ensure correct orientation of the bio-recognition element (e.g. DNA sequence, antibody or enzyme) (Chapman et al., 2000; Gebala and Schuhmann, 2010). Self-assembled layers are often formed by incubation of gold surfaces with solutions of thiolated biomolecules and can contain single molecule (monolayer) or multi component forms where additional complexity is introduced in order to ensure adequate orientation of the receptor and good resistance to surface fouling.

Printed circuit board (PCB) based electrochemical sensors are an attractive option for point-of-care sensing. PCBs allow sensors to be mass manufactured at low cost, detect multiple analytes simultaneously and are a highly versatile platform for diagnostic development (Zhao et al., 2020). Sensors produced on PCB electrodes have been used for the detection of a variety of analytes including DNA (Jolly et al., 2019), glucose (Dutta et al., 2020), hormones (Khan et al., 2017) and cytokines (Moschou et al., 2016), showing the wide the range of biological targets PCB devices can accommodate.

In this paper we report the production of a highly manufacturable binary SAM composed of $1 \mathrm{H}, 1 \mathrm{H}, 2 \mathrm{H}, 2 \mathrm{H}-$ Perfluorodecanethiol (PFDT) on gold sensor surfaces which is then bio-functionalised with ACE2 enzyme. PFDT spontaneously forms a dense amphiphobic SAM on gold surfaces, and reduces surface biofouling (Xu et al., 2017). This is achieved due to the fluorous effect (a well known and well described tendency for fluorine atoms to avoid unfavoured interactions with other elements) (Flynn et al., 2017; Shah, 2018; Wong, 2012). Such a surface resists biofouling from amphiphilic biomolecules and cells. Crucially, biomolecules such as ACE2 that have hydrophobic tail regions for membrane insertion will be able to physisorb into the fluorous layer. Post ACE2 functionalisation and following target sample exposure, this means that there is potential for a small contribution to the signal from hydrophobic biomolecules and general biofouling, but specific interactions between the spike protein and ACE2 will dominate the response. PFDT has been previously used to enhance performance of organic transistors (Cai et al., 2008) and to reversibly organise DNA onto a micro-patterned substrate (Flynn et al., 2017). The presented sensor uses EIS to detect binding from solutions of recombinant SARS-CoV-2 spike protein and 
positive and negative samples of inactivated SARS-CoV-2 from a fully validated molecular standards kit. Salivaomics are diagnostic techniques that analyse and measure saliva samples for markers of immunological, inflammatory, and infectious diseases (Shah, 2018; Wong, 2012) and the sensor plays to this area by being designed to detect live virus in saliva which affords several key advantages over some other approaches: the results can be produced in a label free manner (i.e. there is no need to add a fluorescent or electrochemical label during the assay steps), there is no chance of detecting residual viral RNA post infection ${ }_{2}$ there is no need for up front sample preparation and crucially the sensor has been designed for ease of upscaling and manufacture with two simple production steps: (1) facile SAM formation and (2) ACE2 functionalisation. The model of diabetes test strip manufacture is particularly applicable, where a surface treatment is first applied followed by printing of glucose oxidase and mediator. This methodology replicates this approach by first coating with PFDT and then physisorbing ACE2 which opens up potential for high volume, low cost production within diabetes test strip production facilities. In the work, initial proof of concept work on the assay is demonstrated on a low cost eight working electrode PCB sensor system. However, the assay can be transferred onto even more mass manufacturable platforms such as screen-printed devices or glucose format test strips. Importantly, this would unlock integration with a well-established high volume production environment and lead to a diagnostic with the potential for widespread, rapid, point of need use.

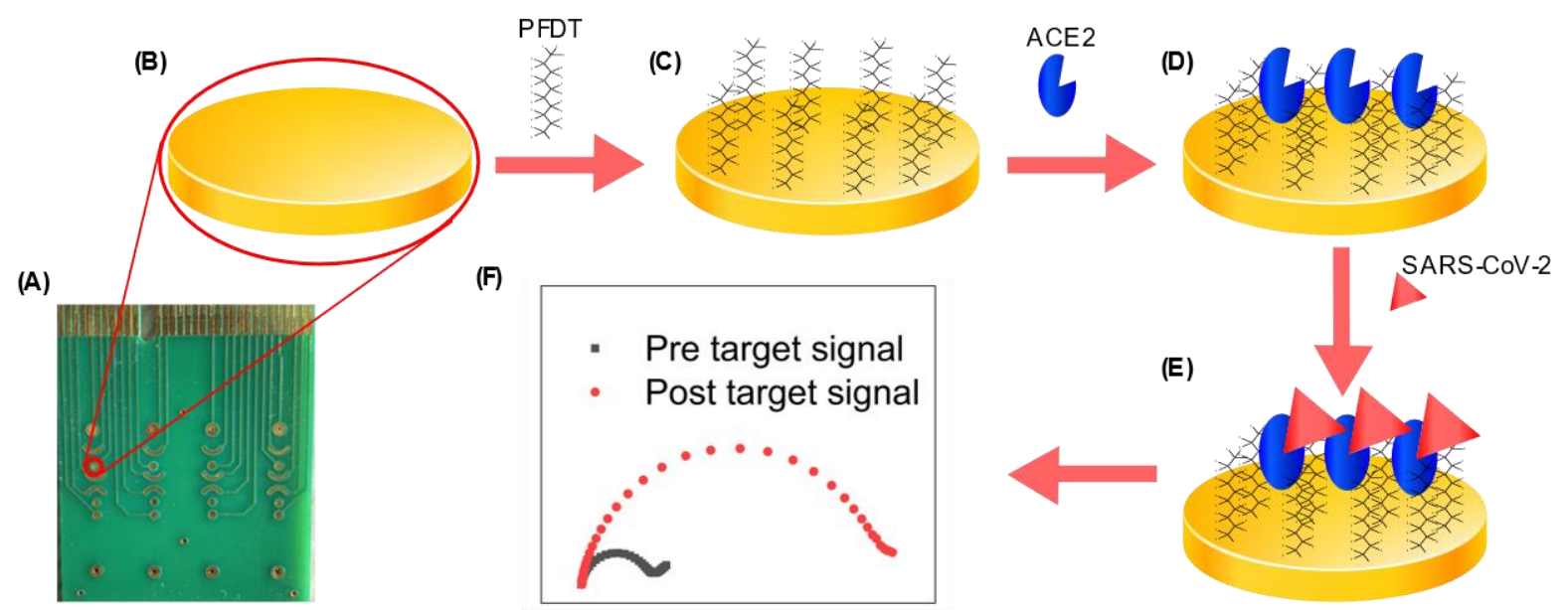

Figure 1. (A) Image of the $8 \times$ Au working electrode PCB based sensor array with on chip Au counter and reference electrodes. (B, C, D, E) Representations of the Au sensor surface in the following states: clean (B), PFDT functionalised and (C) ACE2 functionalised (D) and with binding of SARS-CoV-2 spike protein or inactivated virus (E). (F) Example Nyquist plots showing the signal from an ACE2 functionalised sensor (black) and following exposure to recombinant SARS-CoV-2 spike protein (red). 


\section{Materials and Methods}

\subsection{Chemicals}

$\mathrm{K}_{3}\left[\mathrm{Fe}(\mathrm{CN})_{6}\right], \mathrm{K}_{4}\left[\mathrm{Fe}(\mathrm{CN})_{6}\right], 1 H, 1 H, 2 \mathrm{H}, 2 \mathrm{H}$-perfluorodecanethiol, $\mathrm{KOH}$ and $\mathrm{H}_{2} \mathrm{O}_{2} 30 \%(\mathrm{v} / \mathrm{v})$ were obtained from Sigma Aldrich. Toluene was obtained from Fisher Scientific UK Ltd (Loughborough, UK). Deionised water (5.00 $\mu \mathrm{S} / \mathrm{cm} @ 25^{\circ} \mathrm{C}$ ) was purchased from Scientific Laboratory Supplies Limited (Nottingham, UK). Inactivated SARSCoV-2 and negative control obtained from Randox laboratories Ltd (Crumlin, UK). ACE2 was purchased from Abcam (Cambridge, UK), HRP conjugated spike protein was purchased from The Native Antigen Company (Oxford, UK) and HRP conjugated streptavidin was purchased as part of an IL-6 diagnostics kit from Bio-techne (Abingdon, UK).

\subsection{Preconditioning}

SEP1 BIOTIP multichannel electrode PCB platform (biotip ltd, Bath, UK) were cleaned according to the supplied protocol. This consisted of a 15 -minute submersion in a solution of $50 \mathrm{mM} \mathrm{KOH} \mathrm{in} \mathrm{H}_{2} \mathrm{O}_{2} 30 \%(\mathrm{v} / \mathrm{v}$ ) at room temperature. The PCB was then rinsed with DI water and dried using compressed air. The PCB was then electrochemically cleaned by submerging in $50 \mathrm{mM} \mathrm{KOH} \mathrm{(DI} \mathrm{water} \mathrm{as} \mathrm{solvent)} \mathrm{with} \mathrm{an} \mathrm{external} \mathrm{platinum} \mathrm{counter}$ electrode (Metrohm, Runcorn, UK) and 3M NaCl Ag/AgCl reference electrode (IJ Cambria, Llanelli, UK). Cyclic voltammetry was performed on all working electrodes on the PCB using the following parameters: potential window was -1.2 to $0.6 \mathrm{~V}$, scan rate of $0.1 \mathrm{~V} / \mathrm{s}$ and 15 scans per electrode. The PCB was then rinsed with DI water and dried again using compressed air. All electrochemical measurements were performed using a PalmSens4 potentiostat and the accompanying PSTrace software, both supplied by Palmsens BV (Houten, Netherlands).

\subsection{Fluorous SAM and ACE2 Immobilisation}

The SAM solution was prepared by magnetically stirring toluene and adding PFDT until a 1 mM solution was formed. Stirring aids in dispersing the PFDT throughout the solution. Fluorocarbons can have low miscibility in organic solvents and have a propensity for self-interaction forming separate phases via the fluorous effect (Cametti et al., 2012; Flynn et al., 2017; Gladysz et al., 2004). The PCBs were orientated horizontally in a small glass petri dish and the PFDT solution added to cover the PCB. The PCBs were incubated overnight at room temperature, then rinsed with DI water (10 second water bottle flow per electrode) and dried with compressed air. All work with toluene was performed in a suitable fume hood with proper halogenated solvent waste disposal routes.

ACE2 was diluted from stock in $1 \mathrm{x}$ PBS to $1 \mu \mathrm{g} / \mathrm{ml}$ and a10 $\mu \mathrm{L}$ aliquot was applied to each working electrode on the PCB and left to incubate for 1 hour at room temperature. Following incubation, the PCBs were rinsed with 1 $\mathrm{x}$ PBS (10 second water bottle flow per electrode) and dried with compressed air.

\subsection{Protein Target Detection}

To investigate evidence of specific binding between ligand (ACE2) and protein (HRP conjugated SARS-CoV-2 spike protein) a series of dilutions of the positive control HRP conjugated SARS-CoV-2 spike protein and negative controls of similar sized proteins (HRP conjugated streptavidin and IL-6) were incubated at room temperature for 30 minutes on the PCB sensor arrays with rinsing with 1 x PBS (10 seconds water bottle flow per electrode) and EIS measurements between each concentration incubation. HRP conjugated SARS-CoV-2 spike protein and IL-6 concentrations used were 1, 10, 50 and $100 \mathrm{ng} / \mathrm{ml}$ (all dilutions in $1 \mathrm{x}$ PBS). HRP conjugated streptavidin was obtained as part of an ELISA kit and the concentration was not disclosed. The accompanying instructions recommended a 1:40 dilution for ELISA assays. The series of dilutions used (1:100, 1:75, 1:50, 1:25 and 1:5) were distributed about the 1:40 recommended dilution. 


\subsection{Inactivated Virus Detection}

For detection of inactivated virus, a clinical molecular standards kit for SARS-CoV-2 was purchased. The kit contained positive and negative samples of the virus present in a complex "transport medium" representative of a clinical sample. A series of dilutions of the positive control (inactivated virus + transport medium and human cells) was incubated for 30 mins at room temperature on the PCBs. The concentrations used were $10^{2}, 10^{3}, 10^{4}$, $10^{5}$ and $10^{6} \mathrm{dC} / \mathrm{ml}$ (digital copies per $\mathrm{ml}$ ). Due to small volume of solutions provided, the negative control (transport medium + human cells) was incubated twice for 30 minutes at room temperature. Room temperature incubations were chosen to replicate the operational environmental conditions likely required for a diagnostic device. The PCBs were rinsed with 1xPBS (10 seconds wash bottle flow per electrode) and EIS measurements performed between each incubation.

\subsection{EIS Parameters}

All EIS measurements used the following parameters. $E_{\mathrm{ac}}=0.01 \mathrm{~V} \mathrm{rms}, \mathrm{E}_{\mathrm{dc}}=0 \mathrm{~V}$, frequency range $=100 \mathrm{kHz}$ to 1 $\mathrm{Hz}$ with 50 frequencies at 9.8/decade and measurements were made versus the open circuit potential (OCP). All measurements were obtained using $5 \mathrm{mM} \mathrm{K}_{3}\left[\mathrm{Fe}(\mathrm{CN})_{6}\right] / \mathrm{K}_{4}\left[\mathrm{Fe}(\mathrm{CN})_{6}\right]$ in $1 \times \mathrm{PBS}$.

\section{Results and Discussion}

\subsection{Perfluorocarbon Functionalisation}

Commonly, SAM based electrochemical biosensors feature a probe molecule directly attached to the sensor surface (via covalent bonding, physisorption and chemisorption) and surrounded by a hydrocarbon-based SAM. Less commonly the hydrocarbon SAM is immobilized first, and the biomolecule adsorbed into it via hydrophobic physisorption interactions, which can affect better orientation of the probe biomolecule, increasing the likelihood of receptor-target binding. Such an approach does however have the disadvantage of being a 'weaker' immobilization method than covalent attachment and therefore there is a higher probability of removing some of the biomolecule during incubations and wash steps. In contrast, fluorocarbons offer greatly increased amphiphobicity (hydrophobic and lipophobic character) over hydrocarbons, providing stronger physisorption and anti-biofouling properties (Cametti et al., 2012; Dalvi and Rossky, 2010; Gladysz et al., 2004; Riess, 2005). The ability of fluorocarbons to form a SAM on Au PCB electrode surfaces was investigated (see Figure 2). An overnight incubation of $1 \mathrm{mM}$ PFDT affected an increase in the measured impedance of the electrodes which is evident as a larger $R_{c t}$ semi-circle (SAM) compared to the impedance of the unmodified surface (Clean) in the Nyquist plot (Figure 2A). Quantitatively this could be seen as a mean percentage increase in $\mathrm{R}_{\mathrm{ct}}$ of $928 \%$ with the clean electrodes having a mean $\mathrm{R}_{\mathrm{ct}}=2.5 \mathrm{k} \Omega$ and the $\mathrm{SAM}$ stage $\mathrm{R}_{\mathrm{ct}}=13 \mathrm{k} \Omega$ (Figure $2 \mathrm{~B}$ ).

$$
\Delta \%=\frac{\mathrm{R}_{\mathrm{ct}-\mathrm{After}}-\mathrm{R}_{\mathrm{ct}-\text { Before }}}{\mathrm{R}_{\mathrm{ct}-\text { Before }}}
$$

Percentage signal change was calculated using Equation 1, where $\Delta \%$ is percentage change, $R_{c t-B e f o r e}$ is the $\mathrm{R}_{c t}$ of the initial stage, and $R_{c t-A f t e r}$ is the $\mathrm{R}_{c t}$ of the incubation stage. Significant differences were gauged from the box plots. If the median of one group lies outside the inter quartile range (IQR) of another it is likely there is a significant difference between the groups. In this initial work with a novel sensing modality, inter-device variation had not been optimised and although all devices showed the same trends, the variation meant hypothesis testing their average response was misleading. The box plot (Figure 2B) showed the clean and SAM stages are likely significantly different as the IQR of both groups do not overlap therefore the PFDT layer formed caused a significant increase in the impedance of the electrodes, providing strong evidence for formation of a layer of immobilised PFDT. This is consistent with the well-established process of SAM formation with the SAM molecules attaching to the surface and forming a well-ordered layer at the interface. Such a layer restricts the amount or rate at which the redox active $\mathrm{Fe}(\mathrm{CN})_{6^{3-/ 4-}}$ ions in the measurement buffer can undergo redox reactions, giving rise to an increase in the impedance measurement. In summary, these data showed that a fluorocarbon SAM was successfully formed on the Au PCB electrodes.

\subsection{ACE2 Immobilisation}


A further benefit of the strongly amphiphobic fluorous SAM is that it provides an ideal environment to facilitate hydrophobic interaction of ACE2 biomolecules. To test this, ACE2 protein was incubated in the presence of the electrode SAM. After 1 hour of incubation with $1 \mu \mathrm{g} / \mathrm{ml}$ of ACE2 solution on the SAM functionalized electrodes, a small impedance increase was apparent (Figure 2A). In absolute terms this was a further $2 \mathrm{k} \Omega \mathrm{R}_{\mathrm{ct}}$ increase over the SAM alone (Figure 2B). This was indicative of a sustained interaction between the enzyme and the supporting SAM layer, most likely attributed to the hydrophobic tail of ACE2 inserting into the PFDT layer. The results reveal ACE2 electrodes were significantly different from the clean group but not from the SAM group. This finding was not entirely unexpected as the fluorous SAM had covered a previously clean surface with a densely packed layer resulting in a large impedance change. ACE2 has added to this layer by adsorbing within the fluorous SAM, further blocking the electrode surface. However, the relatively low number of ACE2 molecules in comparison to the fluorous molecules present on the surface and the relatively long distance to the surface accounts for the small relative change in impedance. Despite that, each electrode (except one) showed an increase in impedance after incubating with ACE2, as evident in Figure 2B, validating the use of a box plot to present the data as opposed to methods such as the bar graph which may miss this detail. Having demonstrated successful assembly of the PFDT SAM and having seen evidence of ACE2 incorporation into the SAM structure a series of ligand binding experiments were next undertaken.
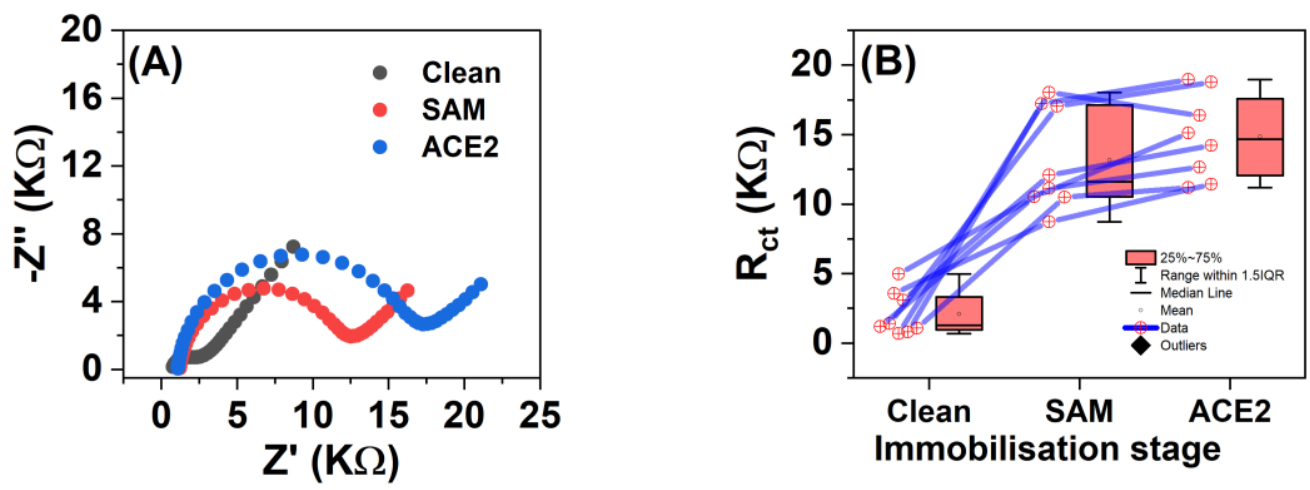

(C)

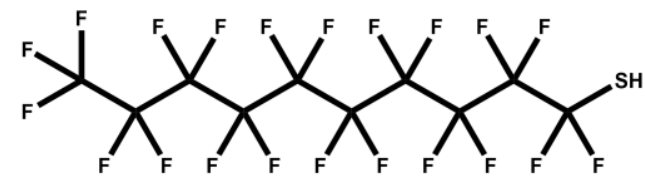

PFDT

Figure 2. (A) Example Nyquist plots from a representative electrode following cleaning (black), PFDT functionalisation (red) and ACE2 incubation (blue). (B) Box plot showing $R_{c t}$ values through the three stages of electrode functionalisation (cleaning, SAM formation and ACE2 immobilisation). (C) Structural formula of PFDT.

\subsection{Detection studies with HRP Conjugated SARS-CoV-2 Spike Protein (positive) and HRP conjugated Streptavidin protein (negative).}

Having confirmed successful immobilisation of ACE2 into the PFDT SAM, HRP conjugated SARS-CoV-2 spike protein (HRP conjugated version employed to enable visual determination of binding - Figure S1) was incubated with the functionalised sensor surface for 30 minutes. The measured impedance for 1, 10, 50 and $100 \mathrm{ng} / \mathrm{ml}$ consistently increased compared to the preceding concentration demonstrating dose dependant behaviour (Figure 3A). The mean percentage change of $\mathrm{R}_{\mathrm{ct}}(\mathrm{n}=4)$ ranged from $96 \%$ at the lowest concentration to $156 \%$ at the highest concentration (Figure 3B, red). This showed the HRP conjugated SARS-CoV-2 spike protein had bound to the PFDT-ACE2 modified sensor. The addition of a diluted series of HRP conjugated streptavidin 
(negative control 1:100,1:75, 1:50, 1:25 and 1:5) allowed for the confirmation of specific binding of HRP conjugated SARS-CoV-2 spike protein. The mean percentage change of $\mathrm{R}_{\mathrm{ct}}(\mathrm{n}=3)$ for the lowest concentration negative control was $-12.8 \%$ and $12 \%$ for the highest (Figure 3B, blue). The negative response thus demonstrated small signal responses to contamination which is further evidenced by the data spreads and values (Figure 3C, blue). All normalised data in these experiments used the PFDT-ACE2 functionalised electrode signal as the normalising factor. There were likely significant differences between the ACE2 and all the positive control concentrations further evidencing strong HRP conjugated SARS-CoV-2 spike protein binding (Figure 3C, red). The negative control experiments were not significantly different along the dilution series indicating weak interaction with the PFDT-ACE2 modified sensor surface. All positive groups are likely significantly different from all negative groups indicated by the median of the negative data lying outside the IQR of the positive groups. Considering this evidence, it was concluded that the HRP conjugated SARS-CoV-2 spike protein successfully and specifically bound to the ACE2 receptor whilst the HRP conjugated streptavidin did not. The signal generated by the negative control was most likely due to a small amount of absorption into the fluorous SAM. The signals are low in comparison to the positive control and appear to saturate at a low level, suggesting that the fluorous SAM layer provided anti-biofouling properties allowing for the positive signal to dominate. It should also be pointed out that the starting concentration of the HRP labelled streptavidin solution was in the region of $1 \mathrm{mg} / \mathrm{mL}$ meaning the dilutions series of negative control protein solutions was significantly more concentrated (at least one order of magnitude) than the HRP conjugated spike protein solutions. The fact that there is strong evidence of specific binding of the positive and comparatively weaker binding of the negative also confirms that ACE2 is physisorbed into the fluorous SAM in significant enough quantity and orientation to bind the target ligand. If ACE2 was bound in an unfavourable orientation, ligand access to receptor binding sites would have been hindered and greatly reduced the positive signal. Critically, it was also observed (Figure S2) that binding efficiency was significantly reduced when using the shorter eight carbon octane-thiol and longer eleven chain undecanethiol. Additionally, it was not possible to replicate these results with a hydrocarbon SAM composed of decanethiol, showing the importance of the PFDT layer in establishing the sensor architecture. This further indicates that the strong amphiphobic character of the PFDT layer produces an adsorption mechanism responsible for the sensor behaviour. Since an HRP label was employed for the positive protein sample it was prudent to also use an HRP labelled negative control to account for the potential of HRP to contribute to the binding signal. HRP conjugated SARS-CoV-2 spike protein is approximately $154 \mathrm{kDa}$ and HRP conjugated streptavidin is approximately $104 \mathrm{kDa}$. The two proteins were thus of relative similar size and both containing the HRP label allowed for good comparison between the two. An indication of the Y-axis limit of detection (LOD) was calculated using Equation 2;

$$
\mathrm{Y}_{\mathrm{LOD}}=\mathrm{Y}_{\mathrm{i}}+3 \mathrm{SD}_{\mathrm{i}}
$$

where $Y_{L O D}$ was the limit of detection of the Y-axis parameter (normalized $\mathrm{R}_{\mathrm{ct}}$ ), $Y_{i}$ was the y-intercept value obtained from linear regression of the data and $S D_{i}$ was the accompanying standard deviation of the y-intercept. The value obtained for the normalised $\mathrm{R}_{c t} Y_{L O D}$ for SARS-CoV-2 HRP conjugated spike protein was 2.1. The lowest concentration signal tested was at the threshold of this limit. All other concentrations were above the limit. A limit of detection for the $\mathrm{X}$-axis was also calculated from the linear regressed data $\left(\mathrm{R}^{2}=0.99342\right)$. Using the $Y_{\text {LOD }}$ and the equation of the fitted line gave an $\mathrm{X}_{\mathrm{LOD}}$ of $1.06 \mathrm{ng} / \mathrm{ml}$ for SARS-CoV-2 HRP conjugated spike protein. 

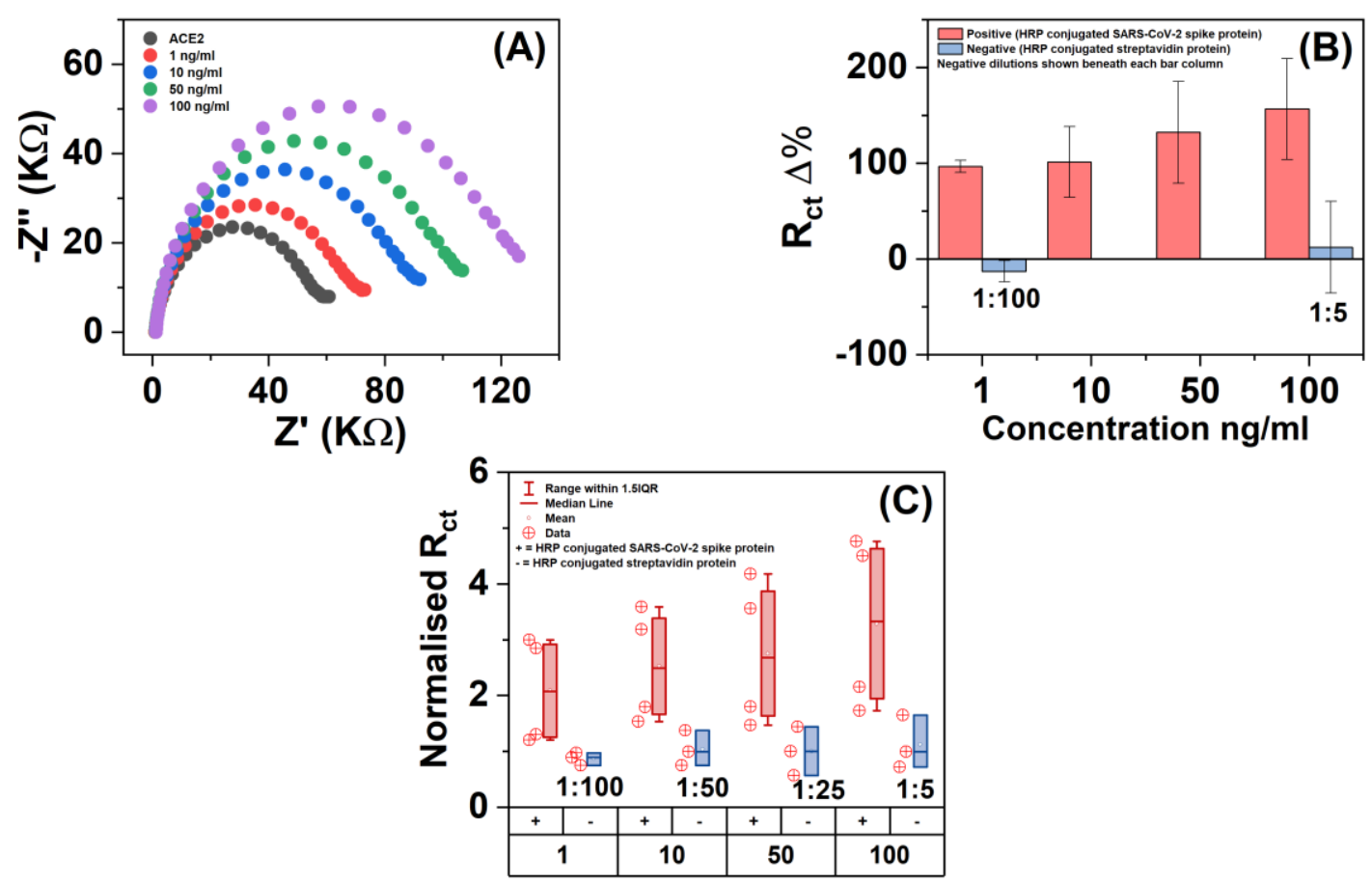

Figure 3. (A) Nyquist plot showing the impedimetric response to increasing HRP conjugated SARS-CoV-2 spike protein. (B) Bar chart showing $\Delta R_{c t} \%$ change in response to addition of HRP conjugated SARS-CoV-2 spike and HRP conjugated streptavidin proteins. (C) Box plot showing normalised $R_{c t}$ values for ACE2 functionalised electrodes versus HRP conjugated SARS-CoV-2 spike protein solutions and HRP conjugated streptavidin solutions.

\subsection{HRP conjugated SARS-CoV-2 Spike Protein (positive) Versus IL-6 (negative).}

A second negative control was investigated using the protein IL-6 (26 kDa) with equal concentrations as used for the positive control $(1,5,10,50,100 \mathrm{ng} / \mathrm{ml})$. IL-6 is a myokine and cytokine common in the human body under normal circumstances, especially after exercise. It has inflammatory and immune effects in a multitude of diseases including bacterial and viral infection. IL-6 has been shown to be present at elevated levels in the 'cytokine storm' which is observed in many advanced cases of COVID-19. This would therefore represent a potential source of noise that could affect specific virus detection and was thus chosen as a negative control. This time, each group used a single PCB array instead of portioning a single board into positive and negative sections. This increased the amount of collected data for both groups from $n=4$ to $n=6$. The HRP conjugated SARS-CoV- 2 spike protein response for increasing concentration was once again seen to sequentially increase (Figure 4A). This was also evident from the $\mathrm{R}_{\mathrm{ct}}$ percentage change (Figure $4 \mathrm{~B}$, red) ranging from $24.4 \%$ at the lowest concentration to $300 \%$ at the highest concentration. This again showed HRP conjugated SARS-CoV-2 spike protein had bound to the PFDT-ACE2 complex. The negative control IL-6 showed smaller mean percentage increases with (Figure 4B, blue). The means were $15 \%$ at the lowest concentration and $39 \%$ at the highest. From the box plots, likely differences between the positive and negative controls of the 1, 50 and $100 \mathrm{ng} / \mathrm{ml}$ concentrations were identified (Figure 4C). The positive spike protein data (Figure 4B and 4C) showed the previously observed increasing dose dependant behaviour. The negative data increased slowly then plateaued in agreement with the small mean percentage changes (Figure 4B) indicating weak IL-6 interactions with the PFDT-ACE2 layer. These results confirmed that the HRP conjugated SARS-CoV-2 spike protein was successfully and specifically detected and that the negative IL-6 signal was suppressed alluding again to weak interaction and anti-biofouling properties arising from the fluorous SAM. It is important to note that the IL- 6 concentrations used were $10^{3}$ to $10^{5}$ times higher than the IL-6 levels detected in COVID-19 patients. Patients that progressed to acute respiratory distress syndrome (ARDS) had a median of $7.39 \mathrm{pg} / \mathrm{mL}$ (Coomes and Haghbayan, 2020; Wu et al., 2020) and patients that died had a median of $11.4 \mathrm{pg} / \mathrm{mL}$ (Coomes and Haghbayan, 2020; Ruan et al., 2020). These values represent serum levels, but IL-6 is present in saliva and has been shown to increase in other diseases (Sri Santosh et al., 2020). We were unable to find data on IL-6 serum vs saliva levels in COVID-19 
patients, but, it is highly likely that IL-6 will be a saliva contaminant and thus a high concentration was used as worst case scenario. This experiment was able to show discrimination with a contamination level far in excess of that seen in clinical COVID-19 samples. The normalized $R_{c t} Y_{L O D}$ was found to be 1.21 (Figure 4D), which was a slight improvement on that reported in the previous section $\left(\mathrm{Y}_{\mathrm{LOD}}=2.1\right)$. Only the $1 \mathrm{ng} / \mathrm{ml}$ concentration data point intersected this limit suggesting that the $1 \mathrm{ng} / \mathrm{ml}$ may not be a reliable value for clear detection. The concentration $\mathrm{X}_{\mathrm{LOD}}$ however was found to be $1.68 \mathrm{ng} / \mathrm{ml}\left(\mathrm{R}^{2}=0.99\right)$.
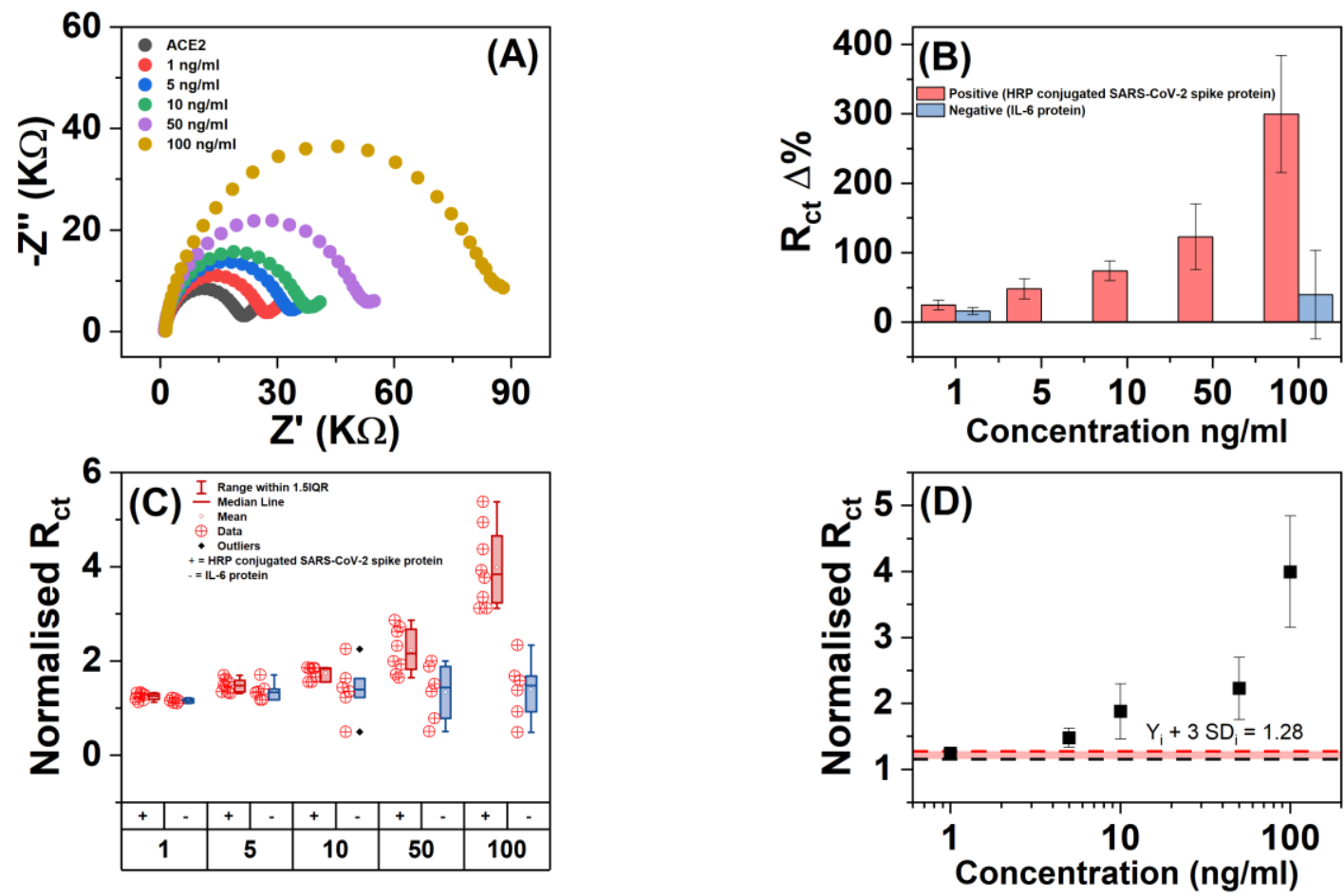

Figure 4. (A) Nyquist plot showing the impedimetric response to increasing HRP conjugated SARS-CoV-2 spike protein. (B) Bar chart showing $\Delta \mathbf{R}_{\mathrm{ct}} \%$ change in response to addition of HRP conjugated SARS-CoV-2 spike and IL-6. (C) Box plot showing normalised Rct values for ACE2 functionalised electrodes versus HRP conjugated SARS-CoV-2 spike protein solutions and IL-6 solutions. (D) Dose response curve for HRP conjugated SARS-CoV-2 spike protein.

\subsection{Inactivated SARS-CoV-2 Whole Virus Detection.}

Having shown that the spike protein ligand was able to specifically bind to the ACE2 receptor in the presence of negative control proteins, the focus changed to whole virus detection. A dilution series of inactivated whole virus $\left(10^{2}, 10^{3}, 10^{4}, 10^{5}\right.$ and $\left.10^{6} \mathrm{dC} / \mathrm{ml}\right)$ was tested against undiluted negative control samples from the same molecular standards kit. The kit is used for the purposes of assay validation for respiratory viruses and all positive and negative samples were suspended in a fluid called "transport medium" which comprised lysed cells and proteins in order to mimic a complex clinical saliva sample. Incubations with inactivated SARS-CoV-2 resulted in a consistently increasing $R_{c t}$ (Figure $5 \mathrm{~A}$ ). The mean percentage change for the positive control $(\mathrm{n}=6)$ ranged from $120 \%$ at the lowest to $232 \%$ at the second highest concentration (Figure 5A, red). The highest concentration saw a decrease from $232 \%$ to $185 \%$. This is possibly due to removal of the virus or virus + ACE2 complex or a subsequent reordering or desorption of the SAM resulting from the presence of large quantities of virus through successive experiments. Similar effects with other high target analyte concentrations have been seen in other work within our group (unpublished data). The negative control contained the same background transport medium plus human cells as found in the positive control but lacked the virus. Actual concentrations and composition of background medium were not provided by the vendor however it was stated that samples were representative of clinical human specimens and viral quantification data was supplied in the form of digital copies per $\mathrm{mL}(\mathrm{dC} / \mathrm{mL})$. The first negative sample $(0 \mathrm{dc} / \mathrm{mL})$ was applied at the same time as the first positive control and underwent the same treatments. This resulted in a mean percentage change of $97 \%$ (Figure 5B, blue). A second negative application was performed at the same time as the second positive. This was equivalent to 60 minutes of exposure to negative control. Only two negative treatments were possible due to the sample 
volume required versus the low volume supplied by the vendor. Both negative responses showed similar mean percentage changes (97\% and $102 \%$ ) Figure S3. This showed that the negative complex sample produced a signal increase but one which saturated immediately. In contrast, the signal from samples containing SARS-CoV2 continued to grow with increasing virus concentration. The normalized data showed that $10^{2}$ and $10^{-3} \mathrm{dC} / \mathrm{ml}^{-}$ concentrations were not significantly different from the negative however $10^{4}, 10^{5}$ and $10^{6} \mathrm{dC} / \mathrm{ml}$ were likely significantly different (Figure 5C). This data showed that the virus was specifically bound to the ACE2 receptor and could be distinguished from the negative at $10^{4} \mathrm{dC} / \mathrm{ml}$ and above. Clinical levels range from $10^{4}$ to $10^{11} \mathrm{RNA}$ copies $/ \mathrm{ml}$, and so is within the distinguishable region presented ${ }^{44-45}$. The performance of the sensor itself was indicated by a normalised $R_{c t} Y_{\text {LOD }}$ of 1.83 (Figure 5D). No data points intersected this limit indicating the lowest $10^{2}$ concentration was a successful detection. The $X_{\text {LOD }}$ was $37.8 \mathrm{dC} / \mathrm{ml}\left(\mathrm{R}^{2}=0.96064\right)$. The sensor therefore had the performance to detect over the entire range tested and with the potential to discriminate lower concentrations if the positive to negative signal ratio is improved upon. The results of testing with inactivated virus were highly compelling; in order to inactivate the virus, the manufacturer had heated it at $65^{\circ} \mathrm{C}$ for 30 mins and then gamma irradiated it so it's structure would have been significantly disrupted. This would most likely reduce the levels of intact spike protein, significantly reducing the ability of the virus to specifically interact with the PFDT-ACE2 layer. Also, the positive and negative virus samples were present in the complex medium first used to culture the cells which produced the virus and therefore bore a similar resemblance to other biological media such as saliva and serum. As mentioned, the fact the kit is used for respiratory assay validation gives confidence these results can be replicated in live samples. The $30 \mathrm{~min}$ incubation times provided compelling signal increases meaning the measurement was relatively fast, especially contrasted to the gold standard technique - nucleic acid amplification detection. Finally, there is considerable room for optimisation of the assay protocol, for example, shortening of the viral incubation step and optimisation of washing procedures to maximise discriminatory power.

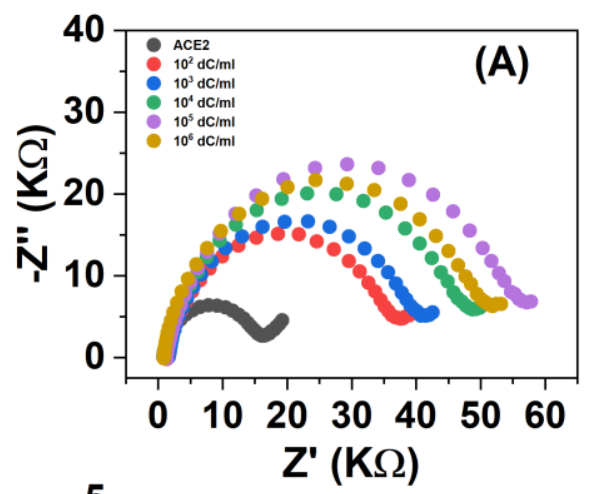

(B)
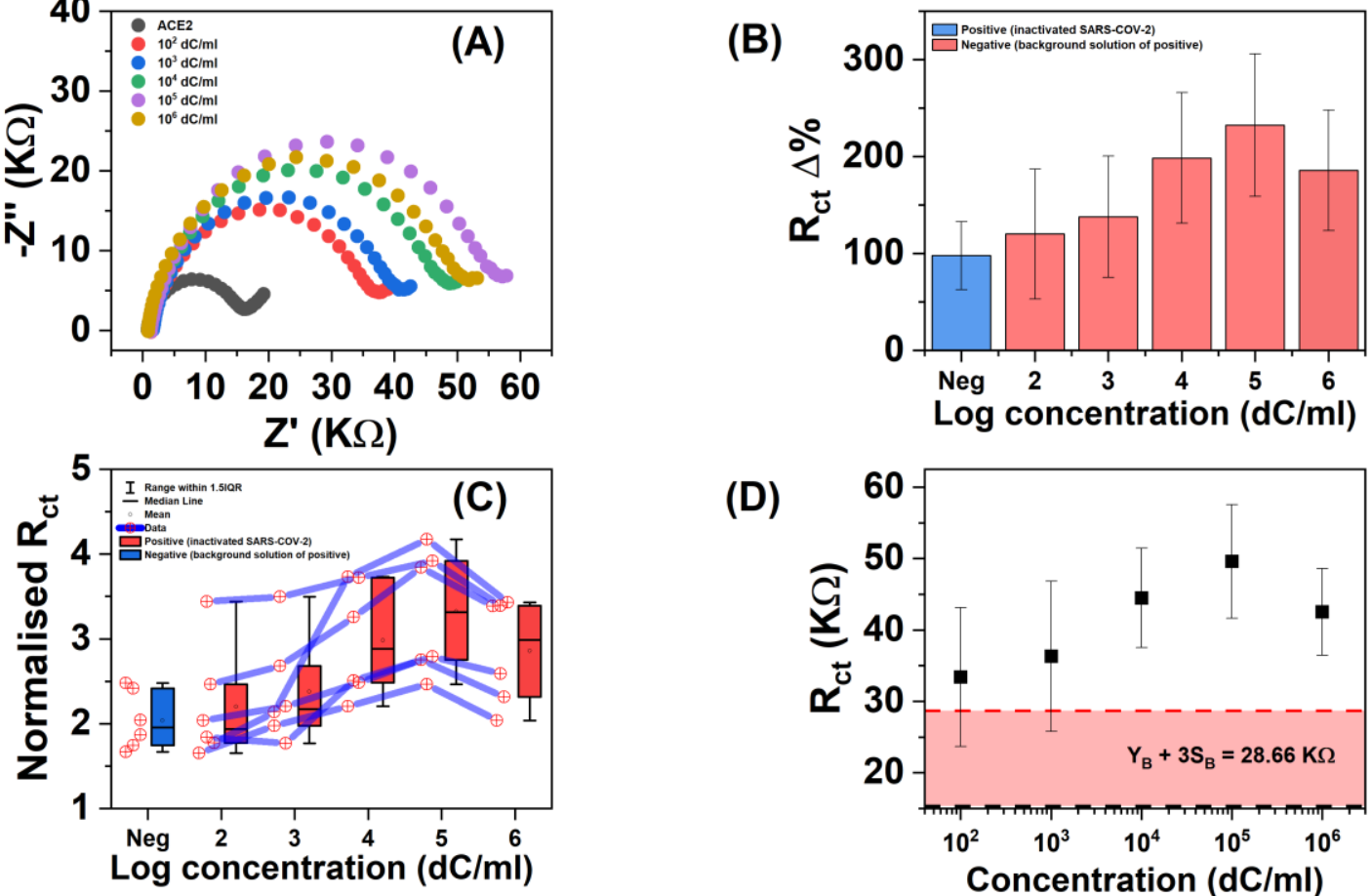

(D)

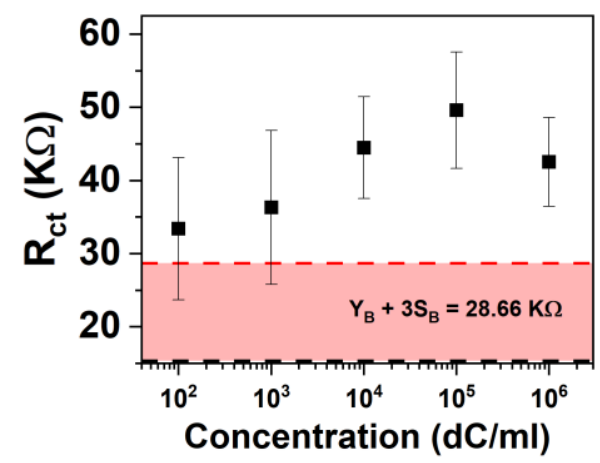

Figure 5. (A) Nyquist plot showing the impedimetric response to increasing concentrations of inactivated SARS-CoV-2 virus. (B) Bar chart showing $\Delta R_{c t} \%$ change in response to addition of negative and positive samples of inactivated SARS-CoV-2. (C) Box plot showing normalised Rct values for ACE2 functionalised electrodes versus positive and negative samples of inactivated SARS-CoV-2. (D) Dose response curve for inactivated SARS-CoV-2. 


\title{
4. Conclusions
}

The preparation and testing of an uncomplicated and straightforwardly produced electrochemical biosensor for SARS-CoV-2 has been demonstrated. The sensor consists of a base SAM composed entirely of PFDT with ACE2 amphiphobically absorbed into the layer. It was possible, using solutions of HRP-conjugated spike protein (positive) and HRP conjugated streptavidin and IL-6 (negatives) to detect the viral spike protein in a sensitive, specific and dose dependant manner. Detection and discrimination of inactivated SARS-CoV-2 virus present in a complex medium (cell culture lysate from a clinical respiratory assay validation kit) was demonstrated to confirm the PFDT-ACE2 layer provided the sensitivity, specificity and resistance to biological fouling necessary for a useful biosensor for SARS-CoV-2. The lower signal change and saturation effect from negative samples demonstrated weak interaction with the PFDT-ACE2 layer. The ease with which the sensor can be prepared and the compatibility of the preparation steps with mass manufacturing techniques mean the assay is potentially adoptable on existing commercial biosensor formats at low cost and high volume. This would allow for wide distribution of point of care assays for rapid testing of the population with diagnostics being at the centre of test, track and tracing of contacts, central to efforts to control the COVID-19 pandemic.

\author{
Abbreviations \\ AUTHOR INFORMATION \\ Corresponding Author \\ * Damion K Corrigan (damion.corrigan@strath.ac.uk) \\ Biomedical Engineering \\ Wolfson centre \\ 106 Rottenrow \\ University of Strathclyde \\ G4 0NS
}

PFDT, $1 H, 1 H, 2 H, 2 H$-perfluorodecanethiol; ACE2, Angiotensin converting enzyme 2; IL-6, Interleukin-6; SARS-CoV-2, Severe acute respiratory syndrome coronavirus 2; HRP, Horseradish peroxidase; EIS, Electrochemical impedance spectroscopy; ARDS, Acute respiratory distress syndrome; PCB, Printed circuit board; PBS, Phosphate-buffered saline; SAM, Self-assembled monolayer; Rct, Charge transfer resistance; OCP, open circuit potential; IQR, inter quartile range.

\section{Author Contributions}

The manuscript was written through contributions of all authors. All authors have given approval to the final version of the manuscript.

\section{Funding Sources}

Chief Scientist's Office (Scotland) COVID-19 Rapid Response Call.

\section{Acknowledgements}

VV would like to thank the EngD Medical Devices CDT for his studentship funded by the EPSRC CDT in Biomedical Devices and Health Technologies (EP/L015595/1). DC would like to thank Prof Phil Bartlett and Dr Maciej Sosna for useful discussions on enzyme attachment chemistry and Prof Glenn Burley for useful discussions on fluorous chemistry. AL and SS would like to thank the staff at LifeScan Scotland regarding contributions towards potential system design options.

\section{References}

Bahadır, E.B., Sezgintürk, M.K., 2016. A review on impedimetric biosensors. Artif. Cells Nanomedicine Biotechnol. 44, 248-262. https://doi.org/10.3109/21691401.2014.942456 
Bain, C.D., Troughton, E.B., Tao, Y.T., Evall, J., Whitesides, G.M., Nuzzo, R.G., 1989. Formation of monolayer films by the spontaneous assembly of organic thiols from solution onto gold. J. Am. Chem. Soc. 111, 321-335. https://doi.org/10.1021/ja00183a049

Bard, A., Faulkner, L., 2001. Electrochemical Methods: Fundamentals and Applications, 2nd ed. Wiley.

Blair, E.O., Hannah, S., Vezza, V., Avc1, H., Kocagoz, T., Hoskisson, P.A., Güzel, F.D., Corrigan, D.K., 2020. Biologically modified microelectrode sensors provide enhanced sensitivity for detection of nucleic acid sequences from Mycobacterium tuberculosis. Sens. Actuators Rep. 2, 100008. https://doi.org/10.1016/j.snr.2020.100008

Butterworth, A., Blues, E., Williamson, P., Cardona, M., Gray, L., Corrigan, D.K., 2019. SAM Composition and Electrode Roughness Affect Performance of a DNA Biosensor for Antibiotic Resistance. Biosensors 9, 22. https://doi.org/10.3390/bios9010022

Cai, Q.J., Chan-Park, M.B., Zhou, Q., Lu, Z.S., Li, C.M., Ong, B.S., 2008. Self-assembled monolayers mediated charge injection for high performance bottom-contact poly $\left(3,3^{\prime \prime \prime}\right.$-didodecylquaterthiophene $)$ thin-film transistors. Org. Electron. 9, 936-943. https://doi.org/10.1016/j.orgel.2008.06.014

Cametti, M., Crousse, B., Metrangolo, P., Milani, R., Resnati, G., 2012. The fluorous effect in biomolecular applications. Chem. Soc. Rev. 41, 31-42. https://doi.org/10.1039/c1cs15084g

Cesewski, E., Johnson, B.N., 2020. Electrochemical biosensors for pathogen detection. Biosens. Bioelectron. 159, 112214. https://doi.org/10.1016/j.bios.2020.112214

Chapman, R.G., Ostuni, E., Takayama, S., Holmlin, R.E., Yan, L., Whitesides, G.M., 2000. Surveying for Surfaces that Resist the Adsorption of Proteins. J. Am. Chem. Soc. 122, 8303-8304. https://doi.org/10.1021/ja000774f

Coomes, E.A., Haghbayan, H., 2020. Interleukin-6 in COVID-19: A Systematic Review and Meta-Analysis. medRxiv 2020.03.30.20048058. https://doi.org/10.1101/2020.03.30.20048058

Dalvi, V.H., Rossky, P.J., 2010. Molecular origins of fluorocarbon hydrophobicity. Proc. Natl. Acad. Sci. 107, 13603-13607. https://doi.org/10.1073/pnas.0915169107

Dutta, G., Regoutz, A., Moschou, D., 2020. Enzyme-assisted glucose quantification for a painless Lab-on-PCB patch implementation. Biosens. Bioelectron. 167, 112484. https://doi.org/10.1016/j.bios.2020.112484

Flynn, G.E., Withers, J.M., Macias, G., Sperling, J.R., Henry, S.L., Cooper, J.M., Burley, G.A., Clark, A.W., 2017. Reversible DNA micro-patterning using the fluorous effect. Chem. Commun. 53, 3094-3097. https://doi.org/10.1039/C7CC00288B

Gebala, M., Schuhmann, W., 2010. Controlled orientation of DNA in a binary SAM as a key for the successful determination of DNA hybridization by means of electrochemical impedance spectroscopy. Chemphyschem Eur. J. Chem. Phys. Phys. Chem. 11, 2887-2895. https://doi.org/10.1002/cphc.201000210

Gladysz, J.A., Curran, D.P., Horváth, I.T., 2004. Handbook of Fluorous Chemistry. John Wiley \& Sons, Ltd. https://doi.org/10.1002/3527603905

Guo, L.-H., Facci, J.S., McLendon, G., Mosher, R., 1994. Effect of Gold Topography and Surface Pretreatment on the Self-Assembly of Alkanethiol Monolayers. Langmuir 10, 4588-4593. https://doi.org/10.1021/la00024a033

Jiang, H., Sun, A., Venkatesh, A.G., Hall, D.A., 2017. An Audio Jack-Based Electrochemical Impedance Spectroscopy Sensor for Point-of-Care Diagnostics. IEEE Sens. J. 17, 589-597. https://doi.org/10.1109/JSEN.2016.2634530

Jolly, P., Rainbow, J., Regoutz, A., Estrela, P., Moschou, D., 2019. A PNA-based Lab-on-PCB diagnostic platform for rapid and high sensitivity DNA quantification. Biosens. Bioelectron. 123, 244-250. https://doi.org/10.1016/j.bios.2018.09.006

Khan, M.S., Misra, S.K., Wang, Z., Daza, E., Schwartz-Duval, A.S., Kus, J.M., Pan, Debanjan, Pan, Dipanjan, 2017. Paper-Based Analytical Biosensor Chip Designed from Graphene-Nanoplatelet-Amphiphilicdiblock-co-Polymer Composite for Cortisol Detection in Human Saliva. Anal. Chem. 89, 2107-2115. https://doi.org/10.1021/acs.analchem.6b04769

Kim, Y.-R., Bong, S., Kang, Y.-J., Yang, Y., Mahajan, R.K., Kim, J.S., Kim, H., 2010. Electrochemical detection of dopamine in the presence of ascorbic acid using graphene modified electrodes. Biosens. Bioelectron. 25, 2366-2369. https://doi.org/10.1016/j.bios.2010.02.031

Lan, J., Ge, J., Yu, J., Shan, S., Zhou, H., Fan, S., Zhang, Q., Shi, X., Wang, Q., Zhang, L., Wang, X., 2020. Structure of the SARS-CoV-2 spike receptor-binding domain bound to the ACE2 receptor. Nature 581, 215-220. https://doi.org/10.1038/s41586-020-2180-5

Lisdat, F., Schäfer, D., 2008. The use of electrochemical impedance spectroscopy for biosensing. Anal. Bioanal. Chem. 391, 1555. https://doi.org/10.1007/s00216-008-1970-7

Manzano, M., Viezzi, S., Mazerat, S., Marks, R.S., Vidic, J., 2018. Rapid and label-free electrochemical DNA biosensor for detecting hepatitis A virus. Biosens. Bioelectron. 100, 89-95. https://doi.org/10.1016/j.bios.2017.08.043 
Masters, P.S., 2006. The Molecular Biology of Coronaviruses. Adv. Virus Res. 66, $193-292$. https://doi.org/10.1016/S0065-3527(06)66005-3

Mehrotra, P., 2016. Biosensors and their applications - A review. J. Oral Biol. Craniofacial Res. 6, 153-159. https://doi.org/10.1016/j.jobcr.2015.12.002

Moschou, D., Greathead, L., Pantelidis, P., Kelleher, P., Morgan, H., Prodromakis, T., 2016. Amperometric IFN$\gamma$ immunosensors with commercially fabricated PCB sensing electrodes. Biosens. Bioelectron. 86, 805810. https://doi.org/10.1016/j.bios.2016.07.075

Newman, J.D., Setford, S.J., 2006. Enzymatic biosensors. Mol. Biotechnol. 32, $249-268$. https://doi.org/10.1385/MB:32:3:249

Riess, J.G., 2005. Understanding the Fundamentals of Perfluorocarbons and Perfluorocarbon Emulsions Relevant to In Vivo Oxygen Delivery. Artif. Cells Blood Substit. Biotechnol. 33, 47-63. https://doi.org/10.1081/BIO-200046659

Ruan, Q., Yang, K., Wang, W., Jiang, L., Song, J., 2020. Clinical predictors of mortality due to COVID-19 based on an analysis of data of 150 patients from Wuhan, China. Intensive Care Med. 1-3. https://doi.org/10.1007/s00134-020-05991-x

Shah, S., 2018. Salivaomics: The current scenario. J. Oral Maxillofac. Pathol. JOMFP 22, 375-381. https://doi.org/10.4103/jomfp.JOMFP_171_18

Shaibani, P.M., Etayash, H., Naicker, S., Kaur, K., Thundat, T., 2017. Metabolic Study of Cancer Cells Using a pH Sensitive Hydrogel Nanofiber Light Addressable Potentiometric Sensor. ACS Sens. 2, 151-156. https://doi.org/10.1021/acssensors.6b00632

Sri Santosh, T., Parmar, R., Anand, H., Srikanth, K., Saritha, M., 2020. A Review of Salivary Diagnostics and Its Potential Implication in Detection of Covid-19. Cureus 12. https://doi.org/10.7759/cureus. 7708

Tancharoen, C., Sukjee, W., Thepparit, C., Jaimipuk, T., Auewarakul, P., Thitithanyanont, A., Sangma, C., 2019. Electrochemical Biosensor Based on Surface Imprinting for Zika Virus Detection in Serum. ACS Sens. 4, 69-75. https://doi.org/10.1021/acssensors.8b00885

Tong, Y., Li, H., Guan, H., Zhao, J., Majeed, S., Anjum, S., Liang, F., Xu, G., 2013. Electrochemical cholesterol sensor based on carbon nanotube@molecularly imprinted polymer modified ceramic carbon electrode. Biosens. Bioelectron. 47, 553-558. https://doi.org/10.1016/j.bios.2013.03.072

Turner, A.P.F., 2013. Biosensors: sense and sensibility. Chem. Soc. Rev. 42, 3184-3196. https://doi.org/10.1039/c3cs35528d

Warner, F.J., Smith, A.I., Hooper, N.M., Turner, A.J., 2004. Angiotensin-converting enzyme-2: a molecular and cellular perspective. Cell. Mol. Life Sci. CMLS 61, 2704-2713. https://doi.org/10.1007/s00018-0044240-7

Wong, D.T.W., 2012. Salivaomics. J. Am. Dent. Assoc. 1939 143, 19S-24S. https://doi.org/10.14219/jada.archive.2012.0339

World Health Organisation, 2020a. Novel Coronavirus - China [WWW Document]. URL http://www.who.int/csr/don/12-january-2020-novel-coronavirus-china/en/ (accessed 9.23.20).

World Health Organisation, 2020b. Director-General's opening remarks at the media briefing on COVID-19 [WWW Document]. URL https://www.who.int/dg/speeches/detail/who-director-general-s-openingremarks-at-the-media-briefing-on-covid-19---11-march-2020 (accessed 9.23.20).

Wu, C., Chen, X., Cai, Y., Xia, J., Zhou, Xing, Xu, S., Huang, H., Zhang, L., Zhou, Xia, Du, C., Zhang, Y., Song, J., Wang, S., Chao, Y., Yang, Z., Xu, J., Zhou, Xin, Chen, D., Xiong, W., Xu, L., Zhou, F., Jiang, J., Bai, C., Zheng, J., Song, Y., 2020. Risk Factors Associated With Acute Respiratory Distress Syndrome and Death in Patients With Coronavirus Disease 2019 Pneumonia in Wuhan, China. JAMA Intern. Med. 180, 934-943. https://doi.org/10.1001/jamainternmed.2020.0994

Xu, D., Su, Y., Zhao, L., Meng, F., Liu, C., Guan, Y., Zhang, J., Luo, J., 2017. Antibacterial and antifouling properties of a polyurethane surface modified with perfluoroalkyl and silver nanoparticles. J. Biomed. Mater. Res. A 105, 531-538. https://doi.org/10.1002/jbm.a.35929

Yang, Z., Gonzalez-Cortes, A., Jourquin, G., Viré, J.-C., Kauffmann, J.-M., Delplancke, J.-L., 1995. Analytical application of self assembled monolayers on gold electrodes: critical importance of surface pretreatment. Biosens. Bioelectron. 10, 789-795. https://doi.org/10.1016/0956-5663(95)99217-9

Zhao, W., Tian, S., Huang, L., Liu, K., Dong, L., 2020. The review of Lab-on-PCB for biomedical application. ELECTROPHORESIS 41, 1433-1445. https://doi.org/10.1002/elps.201900444 